\title{
Singular Distance Powers of Circuits
}

\author{
Torsten SANDER \\ Technische Universität Clausthal \\ (Communicated by M. Tsuchiya)
}

\begin{abstract}
In this work a precise condition for the singularity of a circuit distance power $C_{n}^{(d)}$ is derived. Namely, either $n$ and $d$ are not relatively prime or the order of 2 in $d+1$ is strictly smaller than in $n$. It is also shown that the simple eigenvalues of circuit distance powers are contained in $\{-2,0,2 d\}$, generalizing a well-known result for circuits. Further, the nullity of $C_{n}^{(d)}$ is calculated.
\end{abstract}

\section{Introduction}

The nullity of a graph, i.e. the multiplicity of the zero eigenvalue in its spectrum, is a property frequently studied in algebraic graph theory. Applications can be found e.g. in chemistry, biology or communication science.

Distance powers of circuits belong to the imporant class of circulant graphs. Such graphs are frequently used for modelling redundancies of communication networks. Since circulant graphs reveal strong symmetries a number of interesting results on them have been found [5]. In this work, we are concerned with the spectral properties of distance powers of circuits. It may not surprise that there exists a simple explicit formula to calculate the eigenvalues of any circulant graph [3]. However, it is not quite obvious under which conditions a certain eigenvalue belongs to the spectrum - not even for the well-studied class of distance powers of circuits.

In this work we derive precise conditions for the singularity of a circuit distance power $C_{n}^{(d)}$, generalize a well-known result on the simple eigenvalues of circuits to distance powers of circuits and finally state the nullity of $C_{n}^{(d)}$.

Some known results on the nullity of graphs include e.g. [11] where the nullity of graphs is studied in terms of certain minimal singular subgraphs (so-called minimal configurations). In [2] it is shown that the nullity of a tree equals the number of its vertices minus the number of vertices covered by a maximum matching. [7] gives a linear time algorithm for the computation of the nullity of a tree. In [10] these results are transferred to unicyclic graphs, yielding a linear time algorithm that can check whether a unicyclic graph is singular. Finally,

Received April 17, 2006; revised November 4, 2006

2000 Mathematics Subject Classification: 05C50 (Primary), 15A18 (Secondary).

Key words: circuit, distance power, singularity, nullity 
[12] proves that the nullity of a unicyclic graph on $n$ vertices may range exactly between 0 and $n-4$.

\section{Preliminaries}

2.1. Basics and notation. For the general basics of graph theory, the reader is referred to sources like [1], [6], or [9].

The $d$-th distance power $G^{(d)}$ of a graph $G$ is formed by taking the vertices of $G$ and adding edges between all vertices whose distance in $G$ is at most $d$.

The adjacency matrix $A(G)=\left(a_{i j}\right)$ of a graph $G$ with vertices $x_{1}, \ldots, x_{n}$ is defined by $a_{i j}=1$ if $x_{i}$ and $x_{j}$ are adjacent and 0 otherwise. The eigenvalues of $G$ are the roots of the characteristic polynomial $\chi(x ; G)=\operatorname{det}(A(G)-x I)$. Since $A(G)$ is symmetric it follows that the eigenvalues of a graph are real and that the multiplicity of a root of $\chi(x ; G)$ equals the dimension of the corresponding eigenspace. We call a graph singular if it has a zero eigenvalue. By the nullity of a graph we denote the multiplicity of the zero eigenvalue.

The foundations of algebraic graph theory are treated in [4], [3] and [8].

Further, let $\bar{z}$ denote the complex conjugate of a complex number $z$ and let $\Re(z)$ be its real part. The sets of positive and non-negative integers are written as $\mathbf{N}$ and $\mathbf{N}_{0}$, respectively.

Let $\operatorname{ord}(p, n)$ denote the order of the prime divisor $p$ with respect to $n$, i.e.

$$
\operatorname{ord}(p, n)=\max \left\{j \in \mathbf{N}_{0}: p^{j} \mid n\right\} .
$$

2.2. Circulant graphs. A matrix in which the $i$-th column vector can be derived from the first column vector by means of a downward rotation by $i-1$ entries is called a circulant matrix. Details on circulant matrices can be found in [5].

A circulant graph is a graph whose adjacency matrix is circulant with respect to a suitable vertex ordering. Note that this definition is invariant under isomorphisms. The spectrum of a circulant graph exhibits very strict structural properties as we will see below.

In the following, we will abbreviate $\omega=e^{\frac{2 \pi i}{n}}$.

THEOREM 1. Let $\left(a_{1}, a_{2}, \ldots, a_{n}\right)^{T}$ be the first column of a real circulant matrix $A$. Then the eigenvalues of $A$ are exactly

$$
\lambda_{r}=\sum_{j=1}^{n} a_{j} \omega^{(j-1) r}, \quad r=0, \ldots, n-1 .
$$

COROLLARY 2. Let $G$ be a circulant graph. Let $\left(0, a_{2}, \ldots, a_{n}\right)^{T}$ be the first column of a circulant adjacency matrix of $G$. Then the eigenvalues of $G$ are exactly

$$
\lambda_{r}=\sum_{j=2}^{n} a_{j} \omega^{(j-1) r}, \quad r=0, \ldots, n-1 .
$$


COROLlARY 3. The eigenvalues of the circuit graph $C_{n}$ are

$$
\lambda_{r}=\omega^{r}+\omega^{-r}=2 \cos \left(\frac{2 \pi r}{n}\right), \quad r=0, \ldots, n-1 .
$$

All eigenvalues of modulus 2 are simple, the other eigenvalues are double.

\section{Distance powers $C_{n}^{(d)}$ of circuits}

In this section we will investigate singularity of the graphs $C_{n}^{(d)}$.

Distance powers of circuits are circulant, therefore we can use Corollary 2 to establish their spectrum.

THEOREM 4. The eigenvalues of $C_{n}^{(d)}$ are exactly

$$
\lambda_{r}=2 \sum_{j=1}^{d} \cos \left(\frac{2 \pi r j}{n}\right), \quad r=0, \ldots, n-1 .
$$

PROOF. Choose a sequential vertex order and apply Corollary 2 to the resulting adjacency matrix of $C_{n}^{(d)}$.

Although we can explicitly compute the eigenvalues of $C_{n}^{(d)}$ it is somewhat intricate to predict the occurrence of a prescribed eigenvalue. In the following, we will develop criteria to determine when $C_{n}^{(d)}$ is singular, i.e. it has a zero eigenvalue.

\subsection{Singularity of $C_{n}^{(d)}$}

THEOREM 5. Let $d<\frac{n}{2}-1$. Then $C_{n}^{(d)}$ is singular if and only if there exist integers $1 \leq r<n$ and $l \in \mathbf{N}_{0}$ such that $d r=\ln$ or $2(d+1) r=(2 l+1) n$.

Proof. We may assume $1 \leq r<n$ (which implies $\omega \neq 1$ ).

Let us abbreviate

$$
S_{n, d, r}=\sum_{j=2}^{d+1} \omega^{j-1}, \quad T_{n, d, r}=\sum_{j=n+1-d}^{n} \omega^{j-1} .
$$

These terms correspond to the sums that occur in Theorem 4 so that $C_{n}^{(d)}$ is singular if and only if there exists some $r \in\{1,2, \ldots, n-1\}$ such that $S_{n, d, r}+T_{n, d, r}=0$.

Now observe that

$$
S_{n, d, r}=\omega \sum_{j=0}^{d-1} \omega^{j}=\omega \frac{\omega^{d}-1}{\omega-1}
$$


and

$$
T_{n, d, r}=\omega \sum_{j=1}^{d} \omega^{n-j}=\omega^{n} \sum_{j=1}^{d} \bar{\omega}^{j}=\bar{\omega} \frac{\bar{\omega}^{d}-1}{\bar{\omega}-1} .
$$

If we write

$$
\Omega=\omega \frac{\omega^{d}-1}{\omega-1}
$$

we see that

$$
S_{n, d, r}+T_{n, d, r}=\Omega+\bar{\Omega} .
$$

Consequently,

$$
S_{n, d, r}+T_{n, d, r}=0 \Leftrightarrow \Re(\Omega)=0 .
$$

Let $\varphi=\frac{2 \pi r}{n}$ so that $\omega=e^{i \varphi}$ (note that $\varphi>0$ ). Substituting $x=\cos \varphi$ and $y=\sin \varphi$ it follows that

$$
\frac{\omega}{\omega-1}=\frac{x+i y}{x-1+i y}=\frac{(x+i y)(x-1-i y)}{(x-1)^{2}+y^{2}}=\frac{1}{2}-\frac{y}{2(1-x)} i .
$$

Also,

$$
\omega^{d}-1=\cos (d \varphi)-1+i \sin (d \varphi) .
$$

Substituting equations (3) and (4) into equation (1) we get

$$
\begin{aligned}
\Re(\Omega) & =\frac{1}{2}(\cos (d \varphi)-1)+\frac{y}{2(1-x)} \sin (d \varphi) \\
& =\frac{1}{2}\left((\cos (d \varphi)-1)+\frac{\sin \varphi}{1-\cos \varphi} \sin (d \varphi)\right) .
\end{aligned}
$$

Thus,

$$
\begin{aligned}
\Re(\Omega)=0 & \Leftrightarrow(\cos (d \varphi)-1)(1-\cos \varphi)+\sin \varphi \sin (d \varphi)=0 \\
& \Leftrightarrow \cos (d \varphi)+\cos \varphi-\cos \varphi \cos (d \varphi)+\sin \varphi \sin (d \varphi)=1 \\
& \Leftrightarrow \cos (d \varphi)+\cos \varphi-\cos ((d+1) \varphi)=1 \\
& \Leftrightarrow \frac{\cos ((d+1) \varphi)-\cos (d \varphi)}{\varphi}=\frac{\cos \varphi-\cos 0}{\varphi}
\end{aligned}
$$

The final equation of (5) allows a geometric interpretation. We require the slopes of two particular secant lines of the cosine function to be equal. In this case, due to the nature of the cosine curve there are only two possible constellations for which the slopes are the same. Either both secant lines must be apart by a nonvanishing multiple of $2 \pi$ or their endpoints, if projected onto the same period of the cosine curve, must be point symmetrical with respect to 
$\frac{\pi}{2}$. The first condition means that $d \varphi=2 \pi l$ and the second yields $(d+1) \varphi=\pi+2 \pi l$. The result now follows by combining (2) and (5).

COROLlary 6. For some $d<\frac{n}{2}-1$ let $C_{n}^{(d)}$ be nonsingular. Then,

$$
\operatorname{gcd}(n, d)=1 .
$$

The order of 2 as a divisor of $n$ and $d+1$ plays a crucial role for the singularity of $C_{n}^{(d)}$ :

COROLLARY 7. Let $d<\frac{n}{2}-1$. Then $C_{n}^{(d)}$ is singular if and only if either

$$
\operatorname{gcd}(n, d)>1
$$

or

$$
\operatorname{gcd}(n, d)=1 \text { and } \operatorname{ord}(2, d+1)<\operatorname{ord}(2, n)
$$

holds.

Proof. Let $C_{n}^{(d)}$ be singular. Assume $\operatorname{gcd}(n, d)=1$. Then by Theorem 5 there exist integers $1 \leq r<n$ and $l \in \mathbf{N}_{0}$ such that $2(d+1) r=(2 l+1) n$. Since $2 l+1$ is odd it follows that $1+\operatorname{ord}(2, d+1) \leq \operatorname{ord}(2, n)$.

For the converse statement we need to consider two cases.

Case 1. Assume that $\operatorname{gcd}(n, d)>1$. Then singularity of $C_{n}^{(d)}$ follows from Corollary 6.

Case 2. Assume that $\operatorname{gcd}(n, d)=1$. Let $\kappa<v$ for $\kappa=\operatorname{ord}(2, d+1)$ and $v=\operatorname{ord}(2, n)$. Then there exist odd integers $u$ and $v$ such that $d+1=2^{\kappa} v$ and $n=2^{v} u$.

Let $a=\operatorname{gcd}(u, v)$ so that $u=a u^{\prime}$ and $v=a v^{\prime}$ for suitable integers $u^{\prime}, v^{\prime}$. Now, if we choose

$$
r=2^{\nu-(\kappa+1)} u^{\prime}, \quad l=\frac{1}{2}\left(v^{\prime}-1\right)
$$

we get $r<n$ by virtue of $2^{\nu-(\kappa+1)}<2^{v}$ and $u^{\prime}<u$. Further,

$$
2(d+1) r=2\left(2^{\kappa} v\right) 2^{\nu-(\kappa+1)} u^{\prime}=2^{\nu} v^{\prime} a u^{\prime}=v^{\prime}\left(2^{v} u\right)=(2 l+1) n
$$

so that by Theorem 5 the result follows.

3.2. Eigenvalue multiplicities. Let us now study eigenvalue multiplicities of distance powers of circuits. The next theorem provides a generalization of the result on eigenvalue multiplicites of circuits (cf. Corollary 3 ) to the class of circuit powers.

THEOREM 8.

1. Let $n$ be odd. Then $\lambda=2 d$ is the only simple eigenvalue of $C_{n}^{(d)}$.

2. Let $n$ be even. If $\lambda$ is a simple eigenvalue of $C_{n}^{(d)}$, then

$$
\lambda \in\{-2,0,2 d\} .
$$


If $\lambda=0$ is a simple eigenvalue of $C_{n}^{(d)}$, then the number $d$ must necessarily be even.

Proof. Let $v=\left(v_{i}\right)$ be an eigenvector for the simple eigenvalue $\lambda$ of $C_{n}^{(d)}$. Let $P$ be the matrix of the automorphism that shifts the vertex numbering modulo $n$ by exactly one. Then $P v$ is also an eigenvector of $C_{n}^{(d)}$ and therefore must be a multiple of $v$ because $\lambda$ is simple. Thus, $\left(v_{1}, \ldots, v_{n-1}, v_{n}\right)^{T}=\mu\left(v_{2}, \ldots, v_{n}, v_{1}\right)^{T}$ for some real number $\mu \neq$ 0 . By repeated substitution we get $v_{1}=\mu v_{2}=\mu^{2} v_{3}=\cdots=\mu^{n-1} v_{n}=\mu^{n} v_{1}$ so that necessarily $\mu^{n}=1$. For all $n \in \mathbf{N}$ we see that $\mu=1$ yields the eigenvector $(1, \ldots, 1)^{T}$, which corresponds to the degree of regularity. For even $n$ we get $\mu=-1$ as a second possible solution and the vector $(1,-1,1,-1, \ldots)^{T}$ as eigenvector candidate. It is readily checked that for even $d$ the candidate is an eigenvector for $\lambda=0$ whereas for odd $d$ it is an eigenvector for $\lambda=-2$.

3.3. The nullity of $C_{n}^{(d)}$. Having determined when a circuit distance power is singular, the next step is to ask for the nullity, i.e. the dimension of the null space or kernel of the graph.

Theorem 9. For given $n, d \in \mathbf{N}$ let $g:=\operatorname{gcd}(n, d)$ and $h:=\operatorname{gcd}(n, d+1)$. Then,

$$
\operatorname{dim} \operatorname{ker} C_{n}^{(d)}=0
$$

for $d \geq\left[\frac{n}{2}\right]$, and

$$
\operatorname{dim} \operatorname{ker} C_{n}^{(d)}= \begin{cases}g-1 & \text { if } \operatorname{ord}(2, d+1) \geq \operatorname{ord}(2, n), \\ g+h-1 & \text { if } \operatorname{ord}(2, d+1)<\operatorname{ord}(2, n) \text { and } 2 \not d, \\ g+h-2 & \text { if } \operatorname{ord}(2, d+1)<\operatorname{ord}(2, n) \text { and } 2 \mid d\end{cases}
$$

for $d<\left[\frac{n}{2}\right]$.

PROOF. For $d \geq\left[\frac{n}{2}\right]$ the graph $C_{n}^{(d)}$ is the complete graph $K_{n}$ so that $C_{n}^{(d)}$ is nonsingular [3]. Let $d<\left[\frac{n}{2}\right]$. According to [5] the column vectors of the matrix

$$
F^{*}=n^{-\frac{1}{2}}\left(\omega^{(i-1)(j-1)}\right)_{i, j=1, \ldots, n} \in \mathbf{C}^{n \times n},
$$

which is the conjugate transpose of the so-called Fourier matrix $F \in \mathbf{C}^{n \times n}$, constitute a complete and universal set of complex eigenvectors for every circulant matrix $M$ of order $n$. In order to determine the nullity of $C_{n}^{(d)}$ we therefore need to count all values $r$ that fulfil the condition of Theorem 5 (note that every such $r$ yields $\lambda_{r}=0$ by Theorem 4 ).

CLAIM 1. There exist exactly $g-1$ values of $r$ (with $1 \leq r<n$ ) such that a solution $l \in \mathbf{N}_{0}$ exists for $d r=\ln$.

Since $\operatorname{gcd}\left(\frac{n}{g}, \frac{d}{g}\right)=1$ we see that the equation $r \frac{d}{g}=l \frac{n}{g}$ has solutions $l \in \mathbf{N}_{0}$ and $1 \leq r<n$ if and only if $r=j \frac{n}{g}$ with integer $j$ satisfying $1 \leq j \leq g-1$. 
Claim 2. Let $\operatorname{ord}(2, d+1)<\operatorname{ord}(2, n)$. Then there exist exactly $h$ values of $r$ (with $1 \leq r<n)$ such that a solution $l^{\prime} \in \mathbf{N}_{0}$ exists for $2(d+1) r=\left(2 l^{\prime}+1\right) n$.

First note that $\frac{n}{h}$ is even so that $\operatorname{gcd}\left(\frac{d+1}{h}, \frac{n}{2 h}\right)=1$. It follows that the equation $r \frac{d+1}{h}=$ $\left(2 l^{\prime}+1\right) \frac{n}{2 h}$ has solutions $l, r \in \mathbf{N}_{0}$ exactly for $r=j^{\prime} \frac{n}{2 h}$ and $2 l^{\prime}+1=j^{\prime} \frac{d+1}{h}$ with odd integer $j^{\prime}$ satisfying $1 \leq j^{\prime}<2 h$ (note that $\frac{d+1}{h}$ is odd).

Claim 3. Let $\operatorname{ord}(2, d+1) \geq \operatorname{ord}(2, n)$. Then $2(d+1) r=\left(2 l^{\prime}+1\right) n$ is not solvable with $1 \leq r<n$ and $l^{\prime} \in \mathbf{N}_{0}$.

Since $\frac{n}{h}$ is odd it follows that $\operatorname{gcd}\left(2 \frac{d+1}{h}, \frac{n}{h}\right)=1$. Consequently, $2 r \frac{d+1}{h}$ is even and $\left(2 l^{\prime}+1\right) \frac{n}{h}$ is odd so that we cannot solve $2 r \frac{d+1}{h}=\left(2 l^{\prime}+1\right) \frac{n}{h}$.

Claim 4. Let $\operatorname{ord}(2, d+1)<\operatorname{ord}(2, n)$. If $d$ is even, then $r=\frac{n}{2}$ is the only integer $1 \leq r<n$ such that simultaneous solutions $l, l^{\prime} \in \mathbf{N}_{0}$ can be found for the equations $d r=\ln$ and $2(d+1) r=\left(2 l^{\prime}+1\right) n$. If $d$ is odd then none such $r$ exists.

From Claims 1 and 2 it follows that necessarily $j_{1} \frac{n}{g}=\left(2 j_{2}+1\right) \frac{n}{2 h}$ for integers $j_{1}, j_{2}$ satisfying $1 \leq j_{1} \leq g-1$ and $0 \leq j_{2} \leq h-1$. Equivalently, $j_{1} 2 h=\left(2 j_{2}+1\right) g$ must hold.

Assume that $d$ is even. Since $n$ is necessarily even it follows that $g$ is even, too. But $\operatorname{gcd}\left(\frac{g}{2}, h\right)=1$ yields that $j_{1}$ must be multiple of $\frac{g}{2}$, hence we obtain valid solutions $j_{1}=\frac{g}{2}$ and $j_{2}=\frac{h-1}{2}$. This yields $r=\frac{n}{2}$.

Assume that $d$ is odd. Then $g$ must be odd as well so that $j_{1} 2 h=\left(2 j_{2}+1\right) g$ cannot be solved.

In order to finish the proof of the theorem it now suffices to combine the above claims.

Obviously, Corollary 7 also follows directly from Theorem 9.

\section{Conclusion}

Based on a well-known formula for the calculation of the spectrum of a circulant graph we have derived explicit conditions for the singularity of a circuit distance power $C_{n}^{(d)}$, depending on the order of 2 as a divisor of $n$ and $d+1$. We have generalized the result that the simple eigenvalues of a circuit are contained in the set $\{-2,2\}$ by showing that the simple eigenvalues of circuit distance powers are contained in $\{-2,0,2 d\}$. Finally, we have presented a theorem on the nullity of $C_{n}^{(d)}$.

ACKNOWLEDGement. The author wishes to thank an unknown referee for his helpful comments.

\section{References}

[ 1 ] BeineKe L. W., and Wilson R. J., Selected topics in graph theory 1-3, Academic Press Inc., London, 1978. 
[ 2 ] Bevis, J. H., Domke, G. S., and Miller V. A., Ranks of trees and grid graphs, J. of Combinatorial Math. and Combinatorial Computing 18 (1995), 109-119.

[3] Biggs, N., Algebraic graph theory, Second Edition, Cambridge Mathematical Library, Cambridge University Press, 1993.

[4 ] Cvetković, D. M., Doob, M., and SAchs H., Spectra of graphs, Theory and application, Second Edition, VEB Deutscher Verlag der Wissenschaften, Berlin, 1982.

[ 5 ] Davis, P. J., Circulant matrices, John Wiley \& Sons, New York-Chichester-Brisbane, 1979, A WileyInterscience Publication, Pure and Applied Mathematics.

[6] Diestel, R, Graph theory, Graduate Texts in Mathematics, Vol. 173, Springer-Verlag, 1959.

[ 7 ] Fricke, G. H., Hedetniemi, S. T., Jacobs D. P., and Trevisan, V., Reducing the adjacency matrix of a tree, Electron. J. Linear Algebra 1 (1996), 34-43.

[ 8 ] Godsil, C., and Royle, G., Algebraic graph theory, Graduate Texts in Mathematics, Vol. 207, Springer, 2001.

[9] Harary, F., Graph theory, Addison-Wesley Publishing Co., Reading, 1969.

[10] LI, J., The determinant of a unicyclic graph's neighborhood matrix, Linear Algebra Appl. 394 (2005), 201216.

[11] ScIRIHA, I. On the construction of graphs of nullity one, Discrete Math. 181 (1998), 193-211.

[12] Xuezhong, T., and LiU B. On the nullity of unicyclic graphs, Linear Algebra Appl. 408 (2005), 212-220.

Present Address:

INSTITUT FÜR MATHEMATIK,

TECHNisChE UNIVERSITÄT ClAUSTHAL,

D-38678 Clausthal-ZELlERFELD, GERMANY.

e-mail: torsten.sander@math.tu-clausthal.de 